Check for updates

Cite this: RSC Adv., 2022, 12, 6772

\title{
Defects in graphene-based heterostructures: topological and geometrical effects
}

\author{
Lei Fan, (D) *a Jin Xu $\mathbb{D}^{a}$ and Yihong Hong ${ }^{b}$
}

The combination of graphene (Gr) and graphene-like materials provides the possibility of using twodimensional (2D) atomic layer building blocks to create unprecedented architectures. The most attractive characteristics are strongly dependent on the various spatial structures, mainly including inplane heterostructures butt-joined at the side of an atomic monolayer through covalent bonds, van der Waals (vdW) heterostructures involving a vertically stacked hybrid structure, and their combinations. Heterostructures can not only overcome the limitations inherent to each material but may also obtain new features by appropriate material combination. However, heterostructures made of vdW force superposition or covalent bond splicing are prone to defects. The introduction of external and internal defects causes local deformation and stress in the material, thereby affecting the physical properties of the material, such as its transport properties and mechanical properties. Therefore, research, utilization and control of these defects are highly critical. This paper reviews the vacancy, topological and geometrical effects of defects in modulating the structures and mechanical responses of Gr-based heterostructures. Moreover, the coupling effects of various defects on the Gr-based heterostructures in multi-physics fields are also discussed. This work aims to improve the understanding of the physical mechanism of defective configurations and their association in low dimensions, so as to realize various configurations and to aid the search for new usages.

Received 7th December 2021 Accepted 31st January 2022

DOI: 10.1039/d1ra08884j

rsc.li/rsc-advances and strong in-plane covalent bonds, ${ }^{\mathbf{1 0 - 1 2}}$ as shown in Fig. 1. Multiple 2D materials of the same or different dimensions can be stacked in a specific way (like Lego bricks) or spliced together. ${ }^{13,14}$

Most graphene/h-BN heterostructures (Gr/h-BN) are limited to a single heterostructure or the inner surface of a vertical structure, regardless of the combinations thereof, which can produce interesting heat-transfer characteristics that depend on the design having a high density of heterogeneous multi-pixel functions.

Hexagonal boron nitride (h-BN) and Gr have a highly homologous configuration but different electrical properties. The integration of $\mathrm{Gr}$ and Gr-like materials can create fascinating heterostructures. Moreover, such heterostructures can not only overcome the intrinsic restrictions of lone materials but may also lead to new performance being acquired by suitable combinations thereof.

h-BN is a dielectric material (band gap $=5.9 \mathrm{eV}$ ) but Gr has no band gap and is considered to be a semiconductor. ${ }^{15,16}$ The band gap of Gr can be opened by the hybridization of the two materials. In addition, vdW heterojunctions provide two additional adjustable degrees of freedom, namely, the number of layers and the angle between layers. ${ }^{17}$ By adjusting these two additional degrees of freedom, the energy band structure and electronic transport characteristics of the system can be changed. ${ }^{18}$ In 2014 , Woods et al. found that the moire fringes
${ }^{a}$ School of Civil Engineering and Architecture, Zhejiang University of Science \& Technology, Hangzhou, PR China.E-mail: fanleigl@foxmail.com; xujin@zust.edu.cn ${ }^{b}$ Shanghai Urban Construction Vocational College, Shanghai, China.E-mail:hyhzju@ $126 . \mathrm{com}$ 
are formed in the $\mathrm{Gr} / \mathrm{h}-\mathrm{BN}$ hybrid structure by rotating it at an angle. Meanwhile, a commensurate-incommensurate transition for a hybrid structure has been realized. ${ }^{19}$ In March 2018, Nature magazine published two findings on Gr superconductivity: two new electronic states were found in the twisted stacking double-layer Gr heterojunction, which could easily realize the transition from an insulator to superconductor. ${ }^{\mathbf{2 0 , 2 1}}$ In February 2019, Nature magazine published three articles, which reported the first independent, experimental observation of the existence of a moiré exciton state in 2D heterostructures by several research groups in the United States. ${ }^{22-24}$ In 2020 , by controlling the twist angle, the "magic angle" characteristics of the Gr-based materials could be extended to other 2D research systems, and the distribution information on the twist angle was also studied. ${ }^{25,26}$ Based on a series of discoveries about the "magic angle", it could be expected to realize the performance of a "superconductor" at room temperature, and this set off a huge wave of interest in such fields as energy, electronics, environmental science, and computer industry. However, when a defect and torsion angle coexist, what effects do they have on the interfacial structure, and phonon and electron transport of a vdW heterojunction? The interaction, load transfer, and structural stability of vdW heterostructures with different spatial structures are affected by stress and local deformation caused by various defects. Due to the difference in stacking mode and twist angle, a coupling effect (defect and physical field) will occur, which will expand or reduce the performance of vdW heterostructures.

It is easy to form various kinds of defects in the process of nucleation and growth, which can then form defect clusters in the process of use, which could ultimately change the physical properties of 2D crystals. Compared with three-dimensional (3D) bulk materials, the physical properties of 2D materials, especially their transport behavior and mechanical properties, are more susceptible to local deformation and stress caused by internal and external defects.

Therefore, how to recognize the characteristics of these defects and how to use and control them are very important. This paper reviews the vacancy, topological, and geometrical effects of defects in modulating the structures and mechanical responses of Gr-based heterostructures. Moreover, the coupling effect of heterojunction defects in the multi-physics field is also discussed in this paper.

\section{Effects of defects in graphene- based heterostructures}

The structural defects of Gr-based materials can be divided into two categories: intrinsic defects, which are composed of non-sp ${ }^{2}$ hybrid carbon atoms in the $2 \mathrm{D}$ structure, ${ }^{27}$ and introduced defects, which are composed of other atoms, such as boron (B), nitrogen $(\mathrm{N})$, or oxygen $(\mathrm{O})$, in the form of covalent bonds between carbon (C) atoms and the $2 \mathrm{D}$ structure. ${ }^{28}$ In addition, graphene vertically stacked heterostructures will form defects when multiple 2D materials are vertically stacked through the weak interactions of the vdW force. Also, graphene planar heterostructures will also form defects when two different atomic monolayers are seamlessly stitched together through covalent bonds. For example, these various defects will affect the electrical, optical, and mechanical properties of Gr-based heterostructures. ${ }^{29}$ The appearance of defects changes the bond length and types of some atomic hybrid orbitals. Changes in the bond length and orbit can change the electrical characteristics of the defect region. Point defects and single-vacancy defects form electron wave scattering centers on the surface, which affect the transmission of electrons and eventually reduce the conductivity. In addition, changes in the bond length and bond angle will affect the anharmonic interactions of atoms in the composite system, and then affect the phonon group velocity and phonon-transport efficiency. ${ }^{30}$ Defects will also produce stress accumulation and promote the out-of-plane displacement, thermal fluctuation, and local bending of Grbased materials. ${ }^{31}$ As a result, the deformation of the structure of Gr-based materials will affect the thermal and electrical transport properties.

\subsection{Vacancy effects of defects in graphene-based heterostructures}

Chemical vapor deposition is considered to be the most effective and high-quality technology for the preparation of graphene and graphene heterojunctions, but chemical vapor deposition still leads to inevitable inherent structural defects with different types and sizes. Vacancy defects may occur in the process of chemical vapor deposition. In addition, the process of stripping graphene or a graphene heterojunction from the metal surface is also prone to causing vacancy defects. By using ion irradiation, the defects of graphene can be effectively controlled to obtain the desired properties. ${ }^{32}$ When an electron beam with appropriate energy bombards the surface of graphene, the carbon atoms on the graphene will break away from the system constraints due to the action of energy. ${ }^{33}$ These carbon atoms will completely leave the surface of graphene, and then form vacancy defects. ${ }^{33}$ Ion irradiation is also suitable for regulating the defects of Gr-based materials so that the required properties can be obtained. ${ }^{34,35}$

Vacancy defects can be divided into single-nanohole, doublenanoholes, and multiple-nanoholes. In 2018, M. Y. $\mathrm{Li}^{36}$ investigated the influence of single-void defects on the heat conduction of the $\mathrm{Gr} / \mathrm{h}-\mathrm{BN}$ interface. Their findings showed that the change in vacancy concentration had a direct relationship with the interfacial thermal conductivity. Using stress analysis and power spectrum analysis, the deterioration of the in-plane mode was shown to be caused by a single vacancy. ${ }^{36}$ Obviously, the loss of an atom will inevitably cause the rupture of the three covalent bonds connected to it. As a result, three dangling bonds are formed. Under the influence of the Jahn-Teller effect, the atoms lost at the interface of a heterojunction will undergo structural re-arrangement and energy variation. O. Y. Bin $^{37}$ showed that the formation energies of the three single-vacancy defects defective-carbon, defective-boron, and defectivenitrogen atoms in the zigzag direction were 6.08, 8.29, and $7.23 \mathrm{eV}$, respectively. The formation energies of the double- 
vacancy defects defective-carbon-carbon, defective-carbonboron, defective-carbon-nitrogen, and defective-nitrogenboron bonds in the zigzag direction were 5.35, 10.25, 8.41, and $10.17 \mathrm{eV}$, respectively. The formation energies $E_{\mathrm{tot}}$ of $\mathrm{SV}_{\alpha}$ and $\mathrm{DV}_{\beta \gamma}$ are assumed to be as follows: ${ }^{37}$

$$
\begin{aligned}
& E\left(\mathrm{SV}_{\alpha}\right)=E_{\text {tot }}^{\mathrm{B}}\left(\mathrm{SV}_{\alpha}\right)-E_{\mathrm{tot}}^{\mathrm{A}}+\mu_{\alpha}, \\
& E\left(\mathrm{DV}_{\beta \gamma}\right)=E_{\text {tot }}^{\mathrm{B}}\left(\mathrm{DV}_{\beta \gamma}\right)-E_{\text {tot }}^{\mathrm{A}}+\mu_{\beta}+\mu_{\gamma}
\end{aligned}
$$

where $E_{\text {tot }}^{\mathrm{A}}$ is the system energies with free-defects, and $E_{\text {tot }}^{\mathrm{B}}$ is the system energies with vacancy defects. When the corresponding atoms i (C, B, or N) are eliminated, their chemical potential can be represented by $\mu_{\mathrm{i}=\alpha, \beta, \gamma}{ }^{37}$ Fig. 2 shows the spatial structure of atoms and the charge profile of the defective $\mathrm{Gr} / \mathrm{h}-\mathrm{BN}$ interface.

For vertical heterostructure, due to the diversity of its spatial structure, this defect can be regarded as a control method for planning the next generation of high-performance electronic

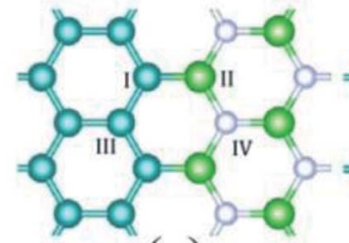

(a)

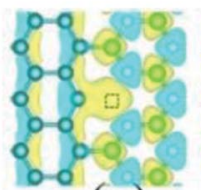

(c)

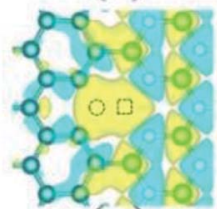

(g)

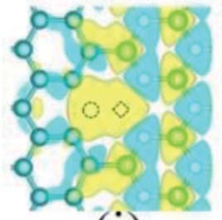

(j)

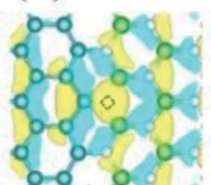

(d)

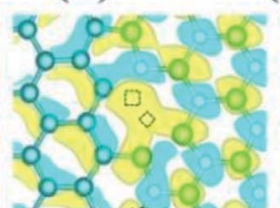

(h)

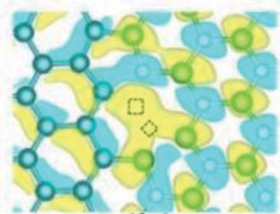

(k)

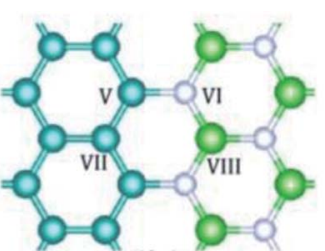

(b)

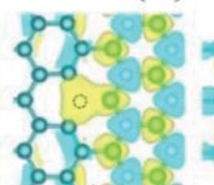

(e)

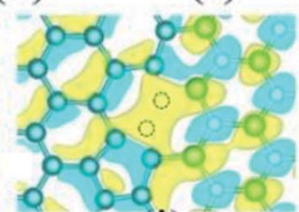

(i)

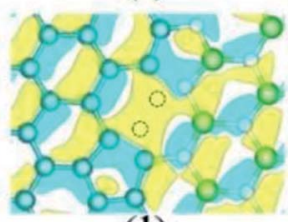

(1)
Fig. 2 Spatial structures of zigzag interfaces with (a) defectivecarbon-boron bonds and (b) defective-carbon-nitrogen bonds. The ground state configuration and charge-transfer profile of singlevacancy: (c) defective boron, (d) defective nitrogen, (e) defective carbon neighboring boron, (f) single-vacancy-carbon neighboring nitrogen in a zigzag interface. The ground state configuration and charge-transfer profile of double-vacancy: (g) defective carbonboron, (h) defective boron-nitrogen and (i) defective carbon-carbon at the defective $\mathrm{Gr} / \mathrm{h}-\mathrm{BN}$ interface with $\mathrm{C}-\mathrm{B}$ bonds in the zigzag direction, (j) double vacancy carbon-nitrogen, (k) double vacancy boron-nitrogen and (l) double vacancy carbon-carbon at the defective $\mathrm{Gr} / \mathrm{h}$-BN interface containing defective-carbon-nitrogen bonds in the zigzag direction. ${ }^{37}$ equipment and vdW heterostructure-enabled nanosensors. M. L. Sun et $a l .{ }^{38}$ employed first-principles calculations to study the spatial configuration and electrical transmission performance of $\mathrm{a} \mathrm{WSe}_{2} / \mathrm{Gr}$ hybrid structure with vacancy nanoholes. Their findings indicated that the performance of a $\mathrm{WSe}_{2} / \mathrm{Gr}$ hybrid structure was greatly transformed when vacancy defects were introduced. A better device property can be acquired by drawing the vacancy defects in the $\mathrm{Gr} / \mathrm{WSe}_{2}$ interface, and then reducing the potential barrier of the interface. ${ }^{38}$ In another work, C. J. Alvarez et al. used the direct growth of both monolayers method to prepare a $\mathrm{MoSe}_{2} / \mathrm{Gr}$ vdW heterostructure. ${ }^{39}$ Moreover, the evolution of the growth processes and their effects on the formation and movement of external defects were studied. ${ }^{39}$ Additionally, in 2018, S. Y. Kim et al. $^{40}$ employed experimental and computational investigations to explore the effect of substrate surface defects on the properties of $2 \mathrm{D}$ vdW structures. Their findings indicate that the imperfection of $\mathrm{Gr}$ have no crucial effect on the configuration or mass of the grown $\mathrm{WS}_{2}$ sheet, but it has the crucial effect on the mutual effect between stacked stratum, thereby affecting the overall properties of the layers. ${ }^{40}$ In 2021, W. Li et al. ${ }^{41}$ investigated the electronic and energy performance of imperfections in a $\mathrm{Gr} / \mathrm{MoS}_{2} \mathrm{vdW}$ heterojunction by DFT theory. The results show that the energy of carbon imperfection was decreased by $0.06-0.54 \mathrm{eV}$, while that of $\mathrm{S}$ imperfection increased by $0.36-0.53 \mathrm{eV}^{41}$ It is worth noting that the influence modes of imperfection on planar heterojunctions and vdW hybrid nanostructures were different; for instance, imperfection in the most momentous section of the planar h-BN/Gr hybrid nanostructure of two monolayers could influence the mechanical performance. The mechanics performance of planar h-BN/Gr nanostructures is affected by the connection method and chemical bond strength. However, vdW heterojunctions are affected not only by chemical bonds, but also by the vdW force. Therefore, under the action of external force and defects, the load transfer and fracture modes of vdW and planar heterojunctions are different.

\subsection{Geometrical effect of defects in graphene-based heterostructures}

When graphene-based heterostructures are deposited and grown on the surface, it usually nucleates in many places at first. Then, the crystals grow separately until they meet. Because the interaction between graphene-based heterostructures and most substrates is weak, and the substrate surface usually has a polycrystalline texture, it is difficult to have the same crystal orientation between the growth nuclei and their crystal domains. Next, when growing single-crystal nuclei that meet at the boundary and form continuous films, topological defects are easy to form in this process. The geometric effects of topological defects on Gr-based heterostructures are shown in two aspects: from the perspective of the geometric shape, and the effects that defects with different geometric shapes have on the properties of Gr-based heterostructures. In 2018, E. E. Kasra demonstrated that two kinds of geometric defects (circular and square nanoholes) are created in $\mathrm{Gr}-\mathrm{BN}-\mathrm{Gr}$ planar heterostructures, ${ }^{42}$ as shown in Fig. 3. Their research showed that 


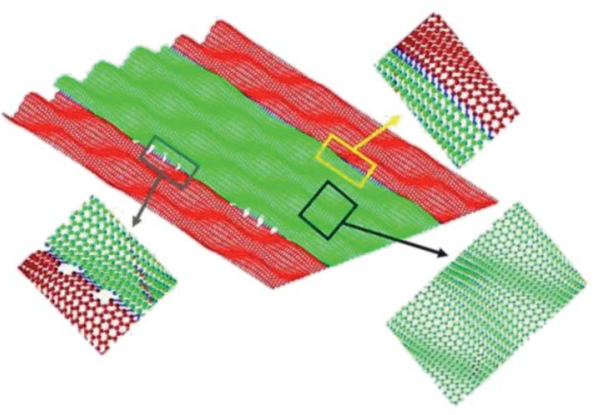

Fig. 3 Two types of geometric imperfection were established in the boundary of the $\mathrm{Gr}-\mathrm{BN}-\mathrm{Gr}$ planar heterostructures. ${ }^{42}$

circular nanoholes have less effect on the mechanical properties of $\mathrm{Gr}-\mathrm{BN}-\mathrm{Gr}$ planar heterostructures, compared with square imperfections. The weaker mechanical properties in the presence of square nanoholes was also attributed to the higher stress concentration and removal of more atoms. ${ }^{42}$ In addition, the orientation of geometric defects (longitudinal and transverse) has different influences on the mechanics performance of $\mathrm{Gr} / \mathrm{h}-\mathrm{BN}$ hybrid nanostructure. This can be due to the different orientation and stretching direction of the imperfections in hybrid nanostructures. ${ }^{43}$ In 2019, L. Fan used molecular dynamics simulation to explore the effect of interlayer $\mathrm{sp}^{3}$ bonds and geometric defects on the mechanical properties of $h$ $\mathrm{BN} / \mathrm{Gr}$ stacked hybrid nanostructures. In this work, the negative effect of rhomboid defects on the mechanical properties of the heterostructure was weaker than that of square defects. ${ }^{\mathbf{4 4}}$

The existence of geometric imperfection is helpful to produce many novel physical phenomena and properties in Grbased heterostructure. In 2020, the influences of geometry on the thermal resistance of $\mathrm{h}-\mathrm{BN} / \mathrm{Gr}$ planar hybrid nanostructures were investigated by MD simulation. ${ }^{45}$ The results showed the addition of interface thermal resistance led to either excessive phonon dispersion, or a decrease in phonon diversion, which was caused by the existence of geometric imperfections in the $\mathrm{Gr} / \mathrm{h}$-BN planar heterostructures. ${ }^{45}$ Besides, the geometric effect of the defect was also closely related to the position of the defect (bonds energies). In another work, a series of geometric imperfections were established in diverse locations of h-BN/Gr planar heterostructures, including h-BN (B-N bonds), Gr (C-C bonds), and the interface (B-C bonds). ${ }^{46}$ The results showed that it was better to place these circular nanopores in the $\mathrm{Gr}$ domain rather than the interface and h-BN domain in order to obtain better ductility and strength. ${ }^{46}$ A key conclusion was drawn that the bonding energy of $\mathrm{C}-\mathrm{C}$ was $607 \mathrm{kcal} \mathrm{mol}^{-1}$, while the bonding energies of B-N and C-B were $389 \mathrm{kcal} \mathrm{mol}^{-1}$ and $448 \mathrm{kcal} \mathrm{mol}^{-1}$, respectively. ${ }^{47}$

Gr-based heterostructures not only depend on the geometric size, defect location, and interface connection, but also the defect structure. The geometric effects of defects, which induce internal stresses in materials, can also lead to geometric deformation effects on structures. Z. G. Song and Z. P. $\mathrm{Xu}^{48}$ investigated the defect-induced geometric effect of Gr; whereby changes in the local bending structure (the shape of the cone and saddle with a positive and negative curvature) of $\mathrm{Gr}$ changed its equivalent tensile and bending stiffness. ${ }^{48}$ In another work, by constructing a polycrystalline Gr model with various topological defects at the grain boundaries (GBs), the relationship between out-of-plane displacement and local mechanical response is discussed. Defects with a positive Gauss curvature had the effect of stiffening and strengthening under indentation tests, while defects with a negative Gauss curvature softened and weakened. ${ }^{49}$ Like with $\mathrm{Gr}$, such a geometric effect of Gr-based heterostructures may be induced by defects. The local out-of-plane deformation (caused by these defects) has a critical influence on the local mechanics response of Gr-based heterostructures.

\subsection{Topological effect of defects in Gr-based heterostructures}

The Stone-Wales (SW) defect is one of the most common topological defects in most planar low-dimensional materials, in which the defect structure retains the number of atoms in the base system without forming any dangling bonds. ${ }^{50}$ The original regular hexagonal network structure will change to an irregular non-hexagonal structure by introducing topology defects. ${ }^{51-53}$ The establishment of SW defects depends on the rotation of carbon-carbon or boron-nitrogen bonds, ${ }^{53}$ as shown in Fig. 4.

The formation energies, and bond angle and dimensions will be changed by introducing the SW imperfection in the $\mathrm{Gr} / \mathrm{h}$ BN planar hybrid nanostructure. ${ }^{53}$ For example, the carboncarbon bonding of defect-free Gr nanostructures $(1.44,1.43 \AA$ ) are equal or smaller than those of pristine $\mathrm{Gr} / \mathrm{h}-\mathrm{BN}$ planar hybrid nanostructures $(1.45,1.43 \AA)$. The carbon-carbon bonding at the SW imperfection $(1.47,1.44 \AA)$ of $\mathrm{Gr} / \mathrm{h}-\mathrm{BN}$ planar hybrid nanostructures is bigger than in the case of defective $\mathrm{Gr}$ (1.36 ̊). In addition, the formation energies of the $\mathrm{Gr} / \mathrm{h}-\mathrm{BN}$ planar heterostructures in the presence of SW defects were $4.05 \mathrm{eV}$, and the range of formation energy followed a certain

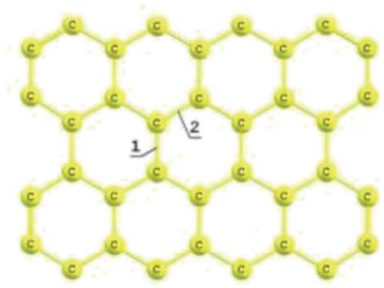

(a)

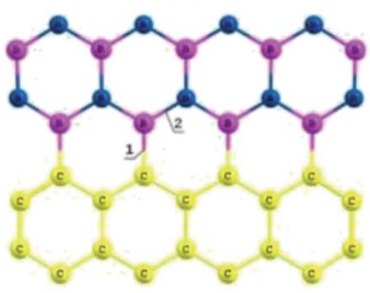

(c)

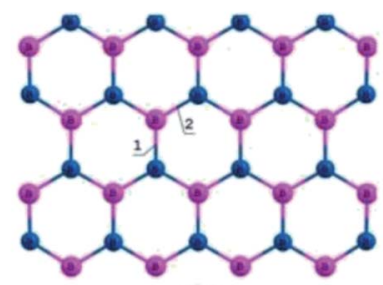

(b)

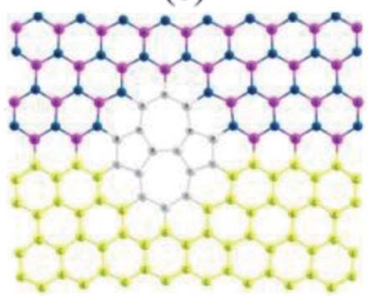

(d)
Fig. 4 Geometric structures of 2D materials: (a) Gr sheet, (b) h-BN sheet, (c) defect-free Gr/h-BN planar heterostructures and (d) $\mathrm{Gr} / \mathrm{h}$ BN planar heterostructures with Stone-Wales defects. ${ }^{53}$ 
rule: $\mathrm{Gr}<\mathrm{Gr} / \mathrm{h}$-BN planar heterostructures $<\mathrm{h}-\mathrm{BN} .{ }^{53}$ Defect formation energies $(E)$ can be expressed by the following formula:

$$
E=E_{\mathrm{SW}}-E_{\text {pristine }}
$$

where $E_{\text {pristine }}$ is the total energy of the pristine nanostructure and $E_{\mathrm{SW}}$ is the total energy of the defective nanostructure in the presence of SW imperfection.

SW defects can be regarded as a dislocation dipole. The initial stress field produced by the dislocation dipole determines the strength of the GBs. GBs are always inevitable. ${ }^{54-58}$ When two materials with different orientations are spliced together, GBs at the interface represent the weak link of the material. These defects will determine the performance of the material. Therefore, it is crucial to know what topological effects the imperfection in defective Gr and other 2D materials will have on their thermal, mechanical, and electrical properties, and what mechanical loads and pressures the defective hybrid materials made of these two $2 \mathrm{D}$ materials can bear.

Liu et al. found that the influence of topological defects on Gr and Gr-based heterostructures (Gr/h-BN) is different. ${ }^{59}$ The thermal conductivity of polycrystalline $\mathrm{Gr}$ will be reduced due to the phonon backscattering caused by atomic defects, ${ }^{60}$ while the thermal conductance of h-BN/Gr hybrid nanostructure will be abnormally enhanced by introducing topological defects at the interface of these two materials. This abnormal enhancement of interfacial thermal conductivity can be attributed to the fact that the stress field is localized due to a mismatch dislocation at the interface and its out-of-plane deformation. ${ }^{59}$ In another work, the thermal transfer efficiency of GBs at the interface of $\mathrm{Gr} / \mathrm{h}-\mathrm{BN}$ heterostructures was reported to not only be related to the direction of heat flow, but also related to the grain misalignment angle. A key conclusion was found that the heattransfer efficiency from h-BN to Gr is lower than that from Gr to h-BN. ${ }^{61}$ In addition, the arrangement of 5-7 disclinations along the GB is very important for the mechanical strength, and the stress concentration at the GB decreases with the increase in disclination density. ${ }^{61}$ R. Daniel demonstrated ${ }^{62}$ that both external and internal disorders are related for vdW hybrid nanostructures composed of Gr and transition metal dichalcogenides. In 2017, P. R. Bandaru ${ }^{63}$ investigated the characteristics and topology of defects on 2D materials surfaces and their influence on charge and energy storage. Their research indicated that topological defects can induce different bond orderings and bond configurations, which then affect the capacitance and the magnitude of the energy storage. The inplane $\mathrm{sp}^{2}$ bonding on each constituent graphene sheet of the graphite involves pendant $\pi$-groups, which may be indirectly involved in charge transfer through weak bonding and adsorption processes. ${ }^{64}$ Furthermore, the effect of topological defects on the electronic characteristic of planar CBN heterostructure was investigated by $\mathrm{S}$. Thomas et $a .^{65}$ The results showed that CBN heterostructures with original defects and SW defects retained the electronic characteristics of direct semiconductors. Interestingly, the single vacancy of C and B makes them metals, which is due to the slight overlapping of the bands at the Fermi levels. ${ }^{65}$

The short linear stacking of topological defects or other types of defects, such as nanoholes or chemical modifications, ${ }^{\mathbf{4 8 , 6 6}}$ will generate stresses that increase with the length of the arrangement within the Gr-based heterostructure. Because of the complex mechanism of failure behavior and a great many influencing factors, including the energy at the GB, initial stress (caused by SW defects), stress gradient and bending angle (caused by the GB), these factors have a certain regulatory function on the properties of the $2 \mathrm{D}$ material, and are different from the behavior in bulk materials. Therefore, how to accurately predict and control the effects of the failure behavior and thermal properties of defective Gr-based heterostructures is a pressing problem which needs to be solved.

\subsection{Quasi-3D effect of defects in graphene-based heterostructures}

Carbon atoms lost during the formation of single-holes and multiple-holes are not necessarily completely separated from Gr. ${ }^{67}$ The carbon atoms may form delocalized atoms, and then migrate on the Gr surface after leaving the original 6-membered ring. ${ }^{68}$ These defects (delocalized atoms) will change the type of orbital hybridization of carbon atoms, in turn resulting in the appearance of $\mathrm{sp}^{3}$ hybrid orbital carbon in $\mathrm{Gr}^{69}$ The introduction of defects in out-of-plane Gr is shown in Fig. 5.

Under the condition of chemical vapor deposition or strong oxidation, the introduction of metal atoms $\mathrm{s}^{71,72}$ or oxygencontaining functional groups ${ }^{73,74}$ on the surfaces of $\mathrm{Gr}$ is inevitable due to the use of metal elements or oxygen-containing oxidants. These heteroatoms are connected with carbon atoms in Gr by strong chemical bonda or weak vdW force, forming out-of-plane heteroatom defects. ${ }^{27}$

Based on the preceding research, Zhang Y. Y. et al. ${ }^{75}$ calculated the influence of $\mathrm{sp}^{3}$ bonding on the physical properties of the bilayer Gr nanostructure. Their findings showed that the $\mathrm{sp}^{3}$ bonding has two opposite effects on the physical properties of the bilayer Gr nanostructure. On the one hand, the $\mathrm{sp}^{3}$ bonding can stimulate a reinforcement effect for the load transfer ability and shear modulus of the bilayer Gr sheets. On the other hand, the tensile stress of the bilayer Gr nanostructure will be reduced due to the local strain induced by $\mathrm{sp}^{3}$ bonding. ${ }^{75}$ In addition, the thermal transport of bilayer and multilayer Gr nanostructures can be controlled by introducing $\mathrm{sp}^{3}$ bonding. ${ }^{76}$ In 2015, Y. F. Gao calculated the influence of $\mathrm{sp}^{3}$ bonding on the thermal transport properties of a Gr-like structure (bilayer boron nitride). ${ }^{77}$ They also that the showed thermal transport properties of bilayered $\mathrm{Gr}$ could be effectively controlled by modifying the interlayer bonding. T. Iwata ${ }^{78}$ indicated the possibility of tuning the thermal conductivity values of bi-Gr and bi-BN by establishing $\mathrm{sp}^{3}$ bonding. Many researchers then began to pay attention to the interlayer bond, especially whether it can bring more novel physical phenomena to the Gr hybrid nanostructure. By studying the in-plane and out-of-plane geometric deformations caused by interlayer bonding, the mechanical model of for a Gr optimal design with the target spatial 

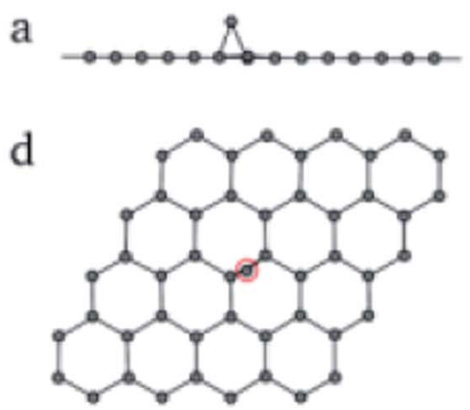

b

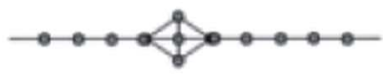

e

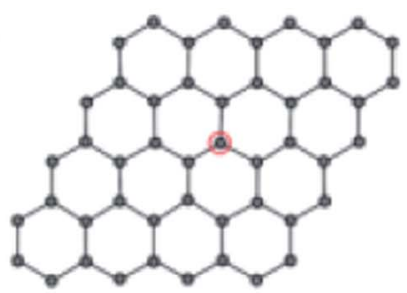

c

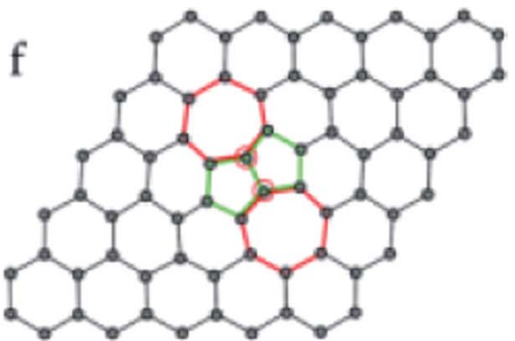

Fig. 5 Carbon atoms defects in Gr: $(a-c)$ space configuration; $(d-f)$ positions of carbon defects. ${ }^{70}$

structure was established by Fan $e t a l$. They expected that the interlayer bonds of Gr-based heterobilayers can play a role in defects for $\mathrm{Gr}$ and 2D materials. ${ }^{79} \mathrm{In} 2020$, W. L. Cai et al. ${ }^{80}$ used experiments and density functional theory to study the effect of defect engineering on carbon black for accelerated Li-S chemistry. In this work, the spatial configurations, bonds structure, and related adsorption energies could be effectively regulated by using defect engineering (see Fig. 6). In another work, the electrocatalytic activity of the carbon architecture in $\mathrm{Li}-\mathrm{S}$ chemistry was manipulated by using a supercritical $\mathrm{CO}_{2}$ foaming technique and defect engineering. ${ }^{81}$ The findings in that study are of great significance for understanding and deciphering the electrocatalytic mechanism of the carbon architecture for the development of advanced Li-S battery applications. ${ }^{81}$

\section{Coupling effect of defects in multiple physical fields}

As mentioned above, defects will produce many novel physical effects, including vacancy, quasi-3D, topological, and geometrical effects and so on. If there are two or more defects in nanostructures at the same time, what coupling effect will the internal field involving the vacancy defect, SW defect, special defect (interlayer $\mathrm{sp}^{3}$ bonds, hydrogenation, functional group and so on) have on the physical properties of Gr-based (a)
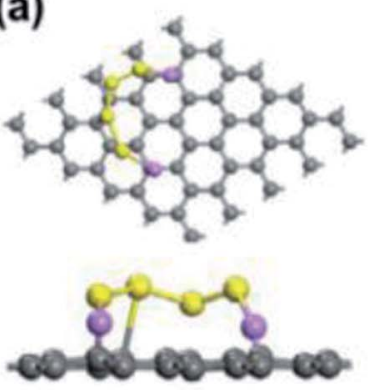

$E_{\text {ads }}=-0.356 \mathrm{eV}$

(d)
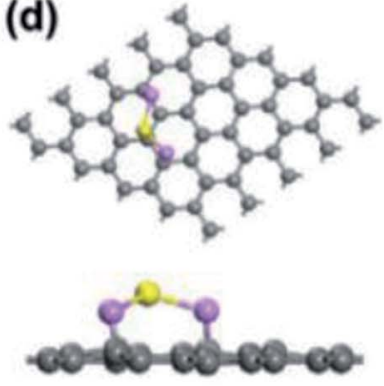

$E_{\text {ads }}=-0.487 \mathrm{eV}$ (b)
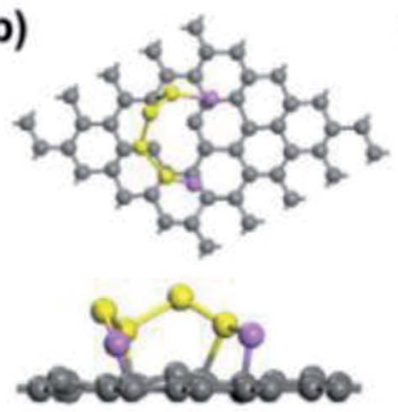

$E_{\text {ads }}=-0.673 \mathrm{eV}$

(e)
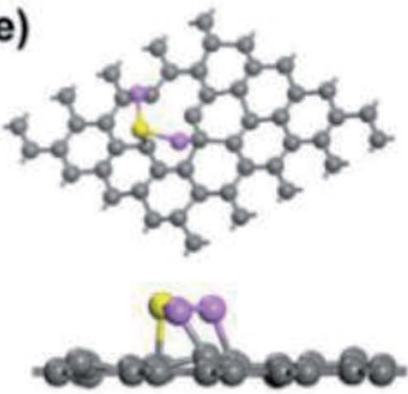

$E_{\text {ads }}=-0.759 \mathrm{eV}$ (c)
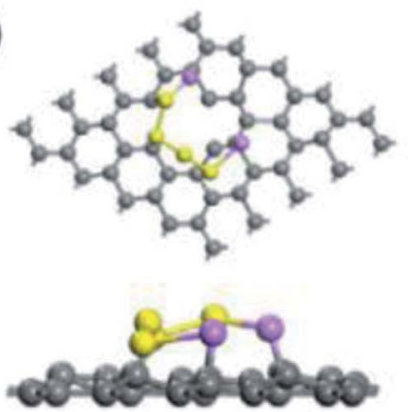

$E_{\text {ads }}=-1.065 \mathrm{eV}$

(f)
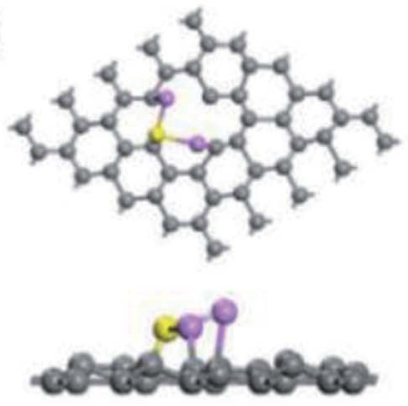

$E_{\text {ads }}=-1.291 \mathrm{eV}$

Fig. 6 Binding configurations and related adsorption energies of $(a-c) L_{2} S_{4}$ and $(d-f) L i_{2} S$ on pristine graphene $((a)$ and $(d))$, monovacancy graphene ((b) and (e)), and divacancy graphene ((c) and (f)). ${ }^{80}$ 
materials? In addition, the effects of the external field ${ }^{82}$ (temperature, strain, electric field, etc.) and internal field on the physical properties of Gr-based heterostructures are also important, because the practical application of Gr-based heterostructures involves the interaction of multiple physical fields, such as heat transfer, vibration, airflow, and compression. In 2014, N. N. $\mathrm{Li}^{83}$ used molecular dynamics simulations to investigate the effects of hydrogenation and the grain boundary on the mechanical properties of polycrystalline Gr. The results revealed that the failure stress of polycrystalline $\mathrm{Gr}$ with a hydrogenated atom was remarkably decreased by the associated shortening effect of bond pre-straining in $\mathrm{sp}^{3}$ atoms and the multiple defective GBs. ${ }^{83}$ In 2017 , E. B. V. Jose ${ }^{84}$ employed first-principles calculations to study the effects of hydrogenation and the grain boundary on the charge transport of hydrogenated polycrystalline graphene, and found that the charge transfer was weakly sensitive to hydrogenation when the adsorbate was confined to the grain boundary. However, the uniform distribution of hydrogen reduced the electron mobility. This difference was due to the formation of hydrogeninduced resonant impurity states. ${ }^{\mathbf{8 4}}$ Therefore, nanoholes, defects, and chemical modification will produce a local stress field, and its amplitude has a certain random distribution in actual material samples. Also, a tunability of the electrical transport of Gr-based materials can be realized through a selective "defect" coupling effect. These studies and related discoveries raised a series of questions for material physics research, and also provided opportunities for the preparation and design of micro-nano materials and structures. In 2018, A. R. $\mathrm{Wei}^{85}$ used a reverse nonequilibrium molecular dynamics method to study the effect of the grain boundary and hydrogenation on the strain-dependent thermal conductivity of hydrogenated polycrystalline graphene under the condition of a strain field. The results showed that the thermal properties of hydrogenated polycrystalline graphene under tension load were related to the average stress in the hydrogenated polycrystalline graphene due to the softening of the phonon modes. ${ }^{85}$ This could be attributed to the fact that both hydrogenation and oxidation will gradually change the material from a honeycomb to a diamond-like structure as result of the hybridization transition from $\mathrm{sp}^{2}$ to $\mathrm{sp}^{3}$, while the introduction of grain boundary will only lead to the distortion of $\mathrm{sp}^{2}$ hybridization. ${ }^{86}$ Also in 2018, using molecular dynamics, the coupling effects of an external field (temperature and strain) and internal field (longitudinal and transverse defects) on the mechanical properties of $\mathrm{Gr} / \mathrm{h}-\mathrm{BN}-\mathrm{Cu}$ heterostructures were investigated by $\mathrm{L}$. Fan et $a l .{ }^{87}$ As a result, atoms near the defect are more likely to escape covalent bond forces when the temperature increases. Moreover, these "defective atoms" are in a semi-active state, and the atoms seem to be applied with an "escape force". ${ }^{87}$ The geometric morphology of the Gr-based heterostructure depends not only on the thermal disturbance of the environment, but also on the defect structure.

Similarly, in 2021, L. Fan et al. used molecular dynamics to study the effect of special defect (interlayer $\mathrm{sp}^{3}$ bonds), intrinsic defects, and the stacking angle on the interfacial thermal transport of $\mathrm{Gr} / \mathrm{h}-\mathrm{BN}$ heterostructures. ${ }^{88}$ Their findings indicated that vdW heterostructures are gradually transformed into quasi-3D nanostructures by introducing interlayer bonding. Furthermore, the interlayer $\mathrm{sp}^{3}$ bond acts as a special defect, and the defect and interlayer bond form a defect amplification effect. Also, in 2020, W. J. Yao et al. ${ }^{89}$ employed molecular dynamics to study the effects of an external (temperature and tension load) and internal (interlayer bonds and nanopores) field on the mechanical properties of $\mathrm{Gr} / \mathrm{h}-\mathrm{BN}$ heterostructures, and found that the atoms close to interlayer bonds and nanopores were more likely to get out of the equilibrium position due to the built-in distortion stress field generated by the coupling effect of temperature and tension loading. ${ }^{89}$ In another work, the mechanical properties of $\mathrm{Gr} / \mathrm{h}$ $\mathrm{BN}$ heterostructures with interlayer bonds and SW defects under the condition of high temperature and load were investigated..$^{90}$ The study findings indicated that the negative effect of interlayer bonding and SW defects on the mechanical properties of the quasi-3D spatial configuration could be accelerated by manipulating multiple physical fields (tensile strain and temperature).

Hence, by studying the response and stability of defective 2D materials under an external field (temperature and load), a physical model for the optimal design of the spatial structure can be established. The quasi-3D effect induced by interlayer bonding and the in-plane stress and structural deformation induced by topological defect may produce more attractive physical effects. These physical effects are particularly significant in high temperature and loading environments, and are different from the behavior in 3D bulk materials, so the physical mechanism is worth exploring.

\section{Conclusion and future prospects}

In practical engineering, Gr-based heterogeneous materials may experience the superposition of physical fields (electricalthermal, thermal-mechanical, and acoustic-structural coupling, etc.), and these physical fields will interact with each other. Gr-based heterogeneous materials inevitably contain various defects, and these defects will affect the properties of Gr-based heterostructures. In the case of multi-field coupling, what effect will the defects have on Gr-based heterostructures?

\subsection{Defect control}

Because the inherent symmetry of the Gr honeycomb lattice is destroyed, point defects induces local states near the Fermi level, which leads to energy splitting at the Dirac point. As a result, the conductivity of Gr gradually decreases, and carrier resonance scattering also occurs. ${ }^{91}$ However, the defects and nanoholes are not all harmful. ${ }^{92}$ The defects improve the semiconductor performance, ion-diffusion coefficient, dispersivity, and reaction activity of Gr-based materials. These defects can be controlled actively, which can then give full play to the advantages of defects in Gr-based materials. Particle irradiation technology can modify and cut Gr-based materials, and change their physical and chemical properties according to the engineering needs. In the process of irradiation, Gr-based 
materials can be damaged by changing the dose, type, energy, and incident angle of the incident particles, thus affecting the performance of Gr-based materials. ${ }^{93-95}$ Particle irradiation (induced defect generation) has aroused great concern in the field of 2D materials. ${ }^{96}$ Hence, it is important to understand the issues and research, including (a) the interaction mechanism between energetic particles and micro-nano targets, (b) the formation theory of structure evolution in Gr-based materials induced by a particle beam, (c) lattice-oriented evolution of the hybrid structure, and (d) the fine control principles of the hybrid structure.

\subsection{Defect coupling and active amplification mechanism}

Many kinds of defects (zero-dimensional defects, onedimensional defects, 2D defects, and introduced defects, etc.) may be formed in the use and preparation of Gr-based materials. It is well known that one type of defect will affect the performance of the system. If there are two or many kinds of defects at the same time, what will happen to the performance of the system? Gr-based materials are more likely to exceed the bound energy limit, and hence can get out of the stable state under the condition of an external field (temperature, strain, and so on). Defects coupling may also produce an active amplification mechanism of the defects due to local deformation and stress (induced by nanoholes or defects).

\subsection{Interlayer defects and structural design}

The diverse interface structures of Gr-based heterostructures, typically including vdW hybrid nanostructures that involve stacking Gr-based nanostructure like building blocks through $\mathrm{vdW}$ force, create the opportunity for diverse and tunable properties. For example, the spatial configuration of Gr-based materials can be optimized by using hydrogen bonds, ion bonds, and polymer cross-linking. These defects not only change the geometric structure (out-of-plane and in-plane geometric deformation) of Gr-like materials, but also affect the load transfer and transport process. In the preparation of $\mathrm{vdW}$ heterostructures, the combination of layers often has a certain design of significance for constructing macroscopic materials. It provides a variety of spatial configurations, and even allows exploring new uses through the regulation of defects.

\section{Abbreviations}

$\begin{array}{ll}\text { vdW } & \text { van der Waals } \\ \text { 2D } & \text { Two-dimensional } \\ \text { 3D } & \text { Three-dimensional } \\ \text { Gr } & \text { Graphene } \\ \text { h-BN } & \text { Hexagonal boron nitride } \\ \text { Gr/h-BN } & \text { Graphene/h-BN heterostructures } \\ \text { GBs } & \text { Grain boundaries } \\ \text { SW } & \text { Stone-Wales } \\ \text { B } & \text { Boron } \\ \text { N } & \text { Nitrogen }\end{array}$

$\begin{array}{ll}\mathrm{O} & \text { Oxygen } \\ \mathrm{C} & \text { Carbon }\end{array}$

\section{Conflicts of interest}

No potential conflict of interest was reported by the authors.

\section{Acknowledgements}

This work was supported by a Doctoral Program of Zhejiang University of Science and Technology (F701104L08).

\section{References}

1 S. K. Bae, H. Kim, Y. B. Lee, et al., Roll-to-roll production of 30-inch graphene films for transparent electrodes, Nat. Nanotechnol., 2010, 5(8), 574-578.

2 M. F. El-Kady, S. Veronica, D. B. Sergey, et al., Laser scribing of high-performance and flexible graphene-based electrochemical capacitors, Science, 2012, 335(6074), 1326-1330.

3 Q. H. Wang, K. Kalantar-Zadeh, A. Kis, et al., Electronics and optoelectronics of two-dimensional transition metal dichalcogenides, Nat. Nanotechnol., 2012, 7(11), 699-712.

4 M. Liu, X. B. Yin, E. Ulin-Avila, et al., A graphene-based broadband optical modulator, Nature, 2011, 474(7349), 6467.

5 K. S. Kim, Y. Zhao, H. Jang, et al., Large-scale pattern growth of graphene films for stretchable transparent electrodes, Nature, 2009, 457(7230), 706-710.

6 A. K. Geim, Graphene: status and prospects, Science, 2009, 324(5934), 1530-1534.

7 A. K. Geim and I. V. Grigorieva, Van der Waals heterostructures, Science, 2013, 499(7459), 419-425.

8 K. S. Novoselov, A. Mishchenko, A. Carvalho, et al., 2D materials and van der Waals heterostructures, Science, 2016, 353(6298), aac9439.

9 Y. Liu, N. O. Weiss, X. D. Duan, et al., Van der Waals heterostructures and devices, Nat. Rev. Mater., 2016, 1(9), 16042.

10 M. Liu, Y. Li, P. Chen, et al., Quasi-freestanding monolayer heterostructure of graphene and hexagonal boron nitride on $\operatorname{Ir}(111)$ with a zigzag boundary, Nano Lett., 2014, 14(11), 6342-6347.

11 G. Y. Lu, T. R. Wu, P. Yang, et al., Synthesis of high-quality graphene and hexagonal boron nitride monolayer in-plane heterostructure on Cu-Ni alloy, Adv. Sci., 2017, 4, 1700076.

12 Z. Liu, L. L. Ma, G. Shi, et al., In-plane heterostructures of graphene and hexagonal boron nitride with controlled domain sizes, Nat. Nanotechnol., 2013, 8(2), 119-124.

13 T. Gao, X. J. Song, H. W. Du, et al., Temperature-triggered chemical switching growth of in-plane and vertically stacked graphene-boron nitride heterostructures, Nat. Commun., 2015, 6, 6835.

14 G. Kim, H. Lim, K. Y. Ma, et al., Catalytic conversion of hexagonal boron nitride to graphene for in-plane heterostructures, Nano Lett., 2015, 15(7), 4769-4775. 
15 N. A. H. Castro, F. Guinea, N. M. R. Peres, et al., The electronic properties of graphene, Rev. Mod. Phys., 2009, 81(1), 109-162.

16 K. Watanabe, T. Taniguchi and H. Kanda, Direct-bandgap properties and evidence for ultraviolet lasing of hexagonal boron nitride single crystal, Nat. Mater., 2004, 3(6), 404-409.

17 R. Frisenda, E. Navarro-Moratalla, P. Gant, et al., Recent progress in the assembly of nanodevices and van der Waals heterostructures by deterministic placement of $2 \mathrm{D}$ materials, Chem. Soc. Rev., 2018, 47(1), 53-68.

18 P. K. Gogoi, Y. C. Lin, R. Senga, et al., Layer rotation-angledependent excitonic absorption in van der Waals heterostructures revealed by electron energy loss spectroscopy, ACS Nano, 2019, 13(8), 9541-9550.

19 C. R. Woods, L. Britnell, A. Eckmann, et al., Commensurateincommensurate transition in graphene on hexagonal boron nitride, Nat. Phys., 2014, 10(6), 451-456.

20 Y. Cao, V. Fatemi, S. A. Fang, et al., Unconventional superconductivity in magic-angle graphene superlattices, Nature, 2018, 556(7699), 43-+.

21 Y. Cao, V. Fatemi, A. Demir, et al., Correlated insulator behaviour at half-filling in magic-angle graphene superlattices, Nature, 2018, 556(7699), 80-+.

22 K. Tran, G. Moody, F. Wu, et al., Evidence for moiré excitons in van der Waals heterostructures, Nature, 2019, 567, 71-75.

23 K. L. Seyler, P. Rivera, H. Yu, et al., Signatures of moirétrapped valley excitons in MoSe2/WSe2 heterobilayers, Nature, 2019, 567, 66-70.

24 C. Jin, E. C. Regan, A. Yan, et al., Observation of moiré excitons in WSe2/WS2 heterostructure superlattices, Nature, 2019, 567, 76-80.

25 Y. Cao, D. Rodan-Legrain, O. Rubies-Bigorda, et al., Tunable correlated states and spin-polarized phases in twisted bilayer-bilayer graphene, Nature, 2020, 583(7815), 221.

26 A. Uri, S. Grover, Y. Cao, et al., Mapping the twist-angle disorder and Landau levels in magic-angle graphene, Nature, 2020, 581, 47-52.

27 S. Sun, C. Y. Wang, M. M. Chen, et al., A novel method to control atomic defects in graphene sheets, by selective surface reactions, Appl. Surf. Sci., 2013, 283, 566-570.

28 S. Sun, C. Y. Wang, M. M. Chen, et al., A method to observe the structure of the interface between mesocarbon microbeads and pitch, J. Colloid Interface Sci., 2014, 426, 206-208.

29 T. Kaneko and T. Ohno, Electron scattering in graphene by defects in underlying h-BN layer: first-principles transport calculations, J. Appl. Phys., 2018, 123(12), 124304.

$30 \mathrm{~W}$. J. Yao and L. Fan, Defects in graphene/h-BN planar heterostructures: insights into the interfacial thermal transport propertie, Nanomaterials, 2021, 11, 500.

31 G. Z. Song and P. Z. Xu, Geometrical effect 'stiffens' graphene membrane at finite vacancy concentrations, Extreme Mech. Lett., 2016, 6, 82-87.

32 S. Kumar, A. Kumar, A. Tripathi, et al., Engineering of electronic properties of single layer graphene by swift heavy ion irradiation, J. Appl. Phys., 2018, 123(16), 161533.
33 J. Hua, Y. Hou, Z. R. Duan, et al., Study on irradiation damage and mechanical properties of graphene, Chin. J. Theor. Appl. Mech., 2016, 48(5), 1080-1087.

34 P. Malinsky, A. Mackova, R. Miksova, et al., Graphene oxide layers modified by light energetic ions, Phys. Chem. Chem. Phys., 2017, 19(16), 10282-10291.

35 L. Fan and W. J. Yao, Regulation and coupling effects on mechanical properties of copper-graphene/h-BN layered heterostructure via ion irradiation, interlayer $\mathrm{sp}^{3}$ bonds and temperature, Mater. Res. Express, 2019, 6, $1050 \mathrm{~b} 1$.

36 M. Y. Li, B. Zheng, K. Duan, et al., Effect of defects on the thermal transport across the graphene/hexagonal boron nitride interface, J. Phys. Chem. C, 2018, 122, 14945-14953.

37 O. Y. Bin, M. Fanchao and S. Jun, Energetics and kinetics of vacancies in monolayer graphene boron nitride heterostructures, 2D Mater., 2014, 1, 035007.

38 M. L. Sun, J. P. Chou, J. Yu, et al., Effects of structural imperfection on the electronic properties of graphene/ $\mathrm{WSe}_{2}$ heterostructures, J. Mater. Chem. C, 2017, 39(5), 10383-10390.

39 C. J. Alvarez, T. D. Minh, A. Marty, et al., Impact of a van der Waals interface on intrinsic and extrinsic defects in an $\mathrm{MoSe}_{2}$ monolayer, Nanotechnology, 2018, 29(42), 425706.

40 S. Y. Kim, J. H. Kim, S. W. Lee, et al., The impact of substrate surface defects on the properties of two-dimensional van der Waals heterostructures, Nanoscale, 2018, 10(40), 1921219219.

41 W. Li, Y. Z. You and J. H. Choi, Vacancy defects in the vertical heterostructures of graphene and $\mathrm{MoS}_{2}$, Surf. Sci., 2021, 707, 121809.

42 E. E. Kasra, S. Sadegh and J. Maisam, Mechanical properties of defective hybrid graphene-boron nitride nanosheets: a molecular dynamics study, Comput. Mater. Sci., 2018, 149, 170-181.

43 E. E. Kasra, S. Sadegh and J. Maisam, Studying the effects of longitudinal and transverse defects on the failure of hybrid graphene-boron nitride sheets: a molecular dynamics simulation, Phys. E, 2018, 104, 71-81.

44 L. Fan and W. J. Yao, Mechanical properties of a G/h-BN heterobilayer nanosheets coupled by interlayer $\mathrm{sp}^{3}$ bonds and defects, Mater. Res. Express, 2019, 6, 095075.

45 E. E. Kasra, S. Sadegh and J. Maisam, Thermal resistance analysis of hybrid graphene-boron nitride nanosheets: the effect of geometry, temperature, size, strain and structural defects, Comput. Mater. Sci., 2020, 174, 109484.

46 L. Fan and W. J. Yao, Effect of defects on mechanical properties of planar h-BN-graphene heterostructure, Mater. Res. Express, 2019, 6, 105613.

47 E. E. Kasra, S. Sadegh and J. Maisam, The mechanical design of hybrid graphene/boron nitride nanotransistors: geometry and interface effects, Solid State Commun., 2018, 270, 82-86.

$48 \mathrm{Z}$. G. Song and Z. P. Xu, Topological defects in twodimensional crystals: the stress buildup and accumulation, J. Appl. Mech., 2014, 81(9), 091004.

49 Z. Song, V. I. Artyukhov, J. Wu, et al., Defect-detriment to graphene strength is concealed by local probe: the 
topological and geometrical effects, ACS Nano, 2015, 9, 401408.

50 S. Thomas and M. A. Zaeem, A new planar BCN lateral heterostructure with outstanding strength and defectmediated superior semiconducting to metallic properties, Phys. Chem. Chem. Phys., 2020, 22, 22066.

51 F. Yin, R. Tarek and B. Cemal, The effect of Stone-Wales defects on the mechanical behavior of graphene nanoribbons, Comput. Mater. Sci., 2016, 124, 142-150.

52 S. P. Wang, J. G. Guo and L. J. Zhou, Influence of StoneWales defects on elastic properties of graphene nanofilms, Phys. E, 2013, 48, 29-35.

53 K. P. Igor and B. P. Konstantin, Stone-Wales defects in graphene-like boron nitride-carbon heterostructures: formation energies, structural properties, and reactivity, Comput. Mater. Sci., 2017, 128, 243-248.

54 A. Reina, X. T. Jia, J. Ho, et al., Large area, few-layer graphene films on arbitrary substrates by chemical vapor deposition, Nano Lett., 2009, 9, 30-35.

55 G. H. Lee, R. C. Cooper, S. J. An, et al., High-strength chemical-vapor deposited graphene and grain boundaries, Science, 2013, 340, 1073-1076.

56 Z. G. Song, V. I. Artyukhov, B. I. Yakobson, et al., Pseudo hallpetch strength reduction in polycrystalline graphene, Nano Lett., 2013, 13, 1829-1833.

57 H. I. Rasool, C. Ophus, W. S. Klug, et al., Measurement of the intrinsic strength of crystalline and polycrystalline graphene, Nat. Commun., 2013, 4, 2811.

58 P. Y. Huang, C. S. Ruiz-Vargas, A. M. van der Zande AM, et al., Grains and grain boundaries in single-layer graphene atomic patchwork quilts, Nature, 2011, 469, 389.

59 X. Liu, G. Zhang and Y. W. Zhang, Topological defects at the graphene/h-BN interface abnormally enhance its thermal conductance, Nano Lett., 2016, 16(8), 4954-4959.

60 A. Bagri, S. P. Kim, R. S. Ruoff, et al., Thermal transport across twin grain boundaries in polycrystalline graphene from nonequilibrium molecular dynamics simulation, Nano Lett., 2011, 11, 3917-3921.

61 Y. F. Li, A. R. Wei, H. Ye, et al., Mechanical and thermal properties of grain boundary in a planar heterostructure of graphene and hexagonal boron nitride, Nanoscale, 2018, 10, 3497.

62 R. Daniel, H. C. Sang, R. P. Rebeca, et al., Disorder in van der Waals heterostructures of 2D materials, Nat. Mater., 2019, 18(6), 541-549.

63 P. R. Bandaru, H. Yamada, R. Narayanan, et al., The role of defects and dimensionality in influencing the charge, capacitance, and energy storage of graphene and 2D materials, Nanotechnol. Rev., 2017, 6(5), 421-433.

64 Q. Li, C. Batchelor-McAuley, R. G. Compton, et al., Electrochemical oxidation of guanine: electrode reaction mechanism and tailoring carbon electrode surfaces to switch between adsorptive and diffusional responses, $J$. Phys. Chem. B, 2010, 114(21), 7423-7428.

65 S. Thomas and M. A. Zaeem, A new planar BCN lateral heterostructure with outstanding strength and defect- mediated superior semiconducting to metallic properties, Phys. Chem. Chem. Phys., 2020, 22(38), 22066-22077.

66 H. I. Rasool, C. Ophus, Z. Zhang, et al., Conserved atomic bonding sequences and strain organization of graphene grain boundaries, Nano Lett., 2014, 14, 7057-7063.

67 P. O. Lehtinen, A. S. Foster, A. Ayuela, et al., Magnetic properties and diffusion of adatoms on a graphene sheet, Phys. Rev. Lett., 2003, 91, 017202.

68 Y. Ma, Simulation of interstitial diffusion in graphite, Phys. Rev. B: Condens. Matter Mater. Phys., 2007, 76, 075419.

69 M. T. Lusk and L. D. Car, Nanoengineering defect structures on graphene, Phys. Rev. Lett., 2008, 100, 175503.

70 B. Florian, K. Jani and V. K. Arkady, Structural defects in graphene, ACS Nano, 2011, 5(1), 26-41.

71 Y. Gan, L. Sun and F. Banhart, One- and two-dimensional diffusion of metal atoms in graphene, Small, 2008, 4(5), 587-591.

72 O. Cretu, A. V. Krasheninnikov, J. A. Rodríguez-Manzo, et al., Migration and localization of metal atoms on strained graphene, Phys. Rev. Lett., 2010, 105(19), 196102.

73 Y. W. Zhu, S. Murali, W. W. Cai, et al., Graphene and graphene oxide: synthesis, properties, and applications, Adv. Mater., 2010, 22(35), 3906-3924.

74 D. A. Dikin, S. Stankovich, E. J. Zimney, et al., Preparation and characterization of graphene oxide paper, Nature, 2007, 448(7152), 457-460.

75 Y. Y. Zhang, C. M. Wang, Y. Cheng, et al., Mechanical properties of bilayer graphene sheets coupled by $\mathrm{sp}^{3}$ bonding, Carbon, 2011, 49, 4511-4517.

76 T. G. Guo, Z. D. Sha, X. J. Liu, et al., Tuning the thermal conductivity of multi-layer graphene with interlayer bonding and tensile strain, Appl. Phys. A: Mater. Sci. Process., 2015, 120, 1275-1281.

77 Y. F. Gao, X. L. Zhang, J. H. Jing, et al., The unexpected nonmonotonic inter-layer bonding dependence of the thermal conductivity of bilayered boron nitride, Nanoscale, 2015, 7, 7143.

$78 \mathrm{~T}$. Iwata and K. Shintani, Reduction of the thermal conductivity of a graphene/hBN heterobilayer via interlayer sp $^{3}$ bonds, Phys. Chem. Chem. Phys., 2018, 20, 5217.

79 L. Fan and W. J. Yao, Effect of interlayer $\mathrm{sp}^{3}$ bonds and nanopores on mechanical properties of vertically-stacked 2D heterostructures, Mater. Res. Express, 2019, 6, 105618.

80 W. L. Cai, Y. Z. Song, Y. T. Fang, et al., Defect engineering on carbon black for accelerated Li-S chemistry, Nano Res., 2020, 13(12), 3315-3320.

81 L. Chen, S. L. Yu, Y. P. Zhang, et al., Manipulating electrocatalytic activity of carbon architecture by supercritical carbon dioxide foaming and defect engineering for Li-S chemistry, J. Power Sources, 2021, 514, 230607.

82 L. Fan and W. J. Yao, Effect of parameter describing pore structure on properties of carbon and graphite materials, Rare Met. Mater. Eng., 2019, 48(8), 2488-2494.

83 N. N. Li, Z. D. Sha, Q. X. Pei, et al., Hydrogenated grain boundaries control the strength and ductility of 
polycrystalline graphene, J. Phys. Chem. C, 2014, 118, 1376913774.

84 E. B. V. Jose, T. F. Jesper, S. David, et al., Grain boundaryinduced variability of charge transport in hydrogenated polycrystalline graphene, 2D Mater., 2017, 4, 025009.

85 A. R. Wei, Q. H. Liu, H. M. Yao, et al., Principles and mechanisms of strain-dependent thermal conductivity of polycrystalline graphene with varying grain sizes and surface hydrogenation, J. Phys. Chem. C, 2018, 122, 1986919879.

86 L. Z. Liu, J. F. Zhang, H. L. Gao, et al., Tailoring physical properties of graphene: effects of hydrogenation, oxidation, and grain boundaries by atomistic simulations, Comput. Mater. Sci., 2016, 112, 527-546.

87 L. Fan and W. J. Yao, Effect of transverse and longitudinal defects on mechanical properties of graphene-h-BN/copper vertically-stacked heterostructure, Comput. Mater. Sci., 2020, 183, 109810.

88 L. Fan and W. J. Yao, Reduction of interfacial thermal transport of bilayer in-plane graphene/hexagonal boron nitride heterostructures via interlayer $\mathrm{sp}^{3}$ bonds, defects and stacking angle, Diamond Relat. Mater., 2021, 118, 108521.

$89 \mathrm{~W}$. J. Yao and L. Fan, Research on the correlation of mechanical properties of $\mathrm{BN}$-graphene-BN/BN verticallystacked nanostructures in the presence of interlayer $\mathrm{sp}^{3}$ bonds and nanopores with temperature, Phys. Chem. Chem. Phys., 2020, 22, 5920.

90 W. J. Yao, L. Fan and Z. P. Zhang, Research on correlation of mechanical behavior of multilayer in-plane graphene/ hexagonal boron nitride heterostructures in the presence of Stone-Wales defects and interlayer $\mathrm{sp}^{3}$ bonds with multiple physical fields, Comput. Mater. Sci., 2020, 185, 109974.

91 N. S. Liu, S. Zhou and J. J. Zhao, Electrical conductance of graphene with point defects, Acta Phys.-Chim. Sin., 2019, 35(10), 1000-6818.

92 W. J. Yao and L. Fan, Advance in properties of graphene and graphene/metal layered composite after irradiation damage, Rare Met. Mater. Eng., 2019, 48(10), 3130-3135.

93 D. Teweldebrhan and A. A. Balandin, Modification of graphene properties due to electron-beam irradiation, Appl. Phys. Lett., 2009, 94(1), 013101.

94 A. V. Krasheninnikov and K. Nordlund, Ion and electron irradiation-induced effects in nanostructured material, $J$. Appl. Phys., 2010, 107(7), 071301.

95 L. Fan and W. J. Yao, Effects of vacancy defects on the mechanical properties of graphene/hexagonal BN superlattice nanoribbons, New Carbon Mater., 2020, 35(2), 165-175.

96 W. J. Yao and L. Fan, The effect of ion irradiation induced defects mechanical properties of graphene/copper layered nanocomposites, Metals, 2019, 9, 733. 